\title{
Caso clínico:
}

Una mujer de 48 años tabaquista de 20 cigarrillos/ día, consulta para dejar de fumar. Había tenido un intento de abandono hacía 2 años, tras el cual había subido $10 \mathrm{Kg}$. por lo que había vuelto a fumar para controlar el peso. En la consulta actual manifiesta gran preocupación por volver a subir de peso y me pregunta que puede hacer para prevenirlo en un nuevo intento de dejar de fumar.

\section{Pregunta}

En pacientes fumadores (población), ¿existe alguna intervención que disminuya la ganancia de peso al dejar de fumar (resultado)?

\section{Estrategias de búsqueda}

Base MEDLINE: Pub med: Filtros metodológicos: Límites: randomized controlled trial, female.

- Términos MesH: smoking cessation weight gain: 46 citas.

- Agregando los siguientes términos MesH a la búsqueda básica, con iguales limites: drug therapy : 7 citas; exercise: 4 citas; diet: 5 citas.

Base OVID: terminos "smoking cessation" "weight gain"

- Journals OVID: 18 citas.

- EBM Reviews Cochrane Database of systematic Reviews (3 er Quarter 2001)

ACP Journal Club desde 1991 hasta agosto 2001, DARE (3 er. Quarter 2001):1 cita.

- EBM Cochrane controlled trials register. (3 er. Quarter 2001): 2 citas.

Los estudios que parecían ser más apropiadas para responder la pregunta son:

1. Danielsson T, Rossner S., Westin A. Open randomized trial of intermittent very low energy diet together with nicotine gum for stopping smoking in women who gained weight in previous attempts to quit. BMJ 1999;19:490-4

2. Marcus Bess H PhD; Albrecht Anna E. RN, MS; King Teresa K $\mathrm{PhD}$; et al. The Efficacy of Exercise as an Aid for Smoking Cessation in Women: A randomized Controlled Trial. Arch Intern Med 1999, 159 (11); 1229-1234

3. Hays J Taylor MD; Hurt, Richard D MD; Rigotti Nancy a md, et al. Sustained- Release Bupropion for Pharmacologic Relapse Prevention after Smoking Cessation: A randomized Controlled Trial. Ann Intern Med 2001,135 (6); 423-433

\section{Cesación tabáquica y aumento de peso}

Alrededor del $80 \%$ de las personas que dejan de fumar aumentan entre 4 y $5 \mathrm{~kg}$ de peso en los 6 meses que siguen a la abstinencia, pudiendo llegar a $11 \mathrm{~kg}$ o más en un $10 \%$ de los casos. El aumento de peso post cesación depende de dos variables: el incremento compensatorio de la ingesta calórica y la disminución del gasto calórico basal que genera la ausencia de nicotina. La nicotina per se, aumenta el consumo basal de energía, motivo por el cual los fumadores tienen un BMI mas bajo que los no fumadores. Esto lleva a que, aun no aumentando el consumo de calorías, las personas que dejan de fumar tienden a subir de peso.

Numerosos estudios evaluaron intervenciones que redujeran la ganancia de peso post cesación, con escasos resultados. Varios de ellos mostraron que la realización de dieta hipocalórica simultáneamente con el tratamiento para dejar de fumar fue contraproducente, aumentando la tasa de recaída tabáquica. La interpretación de este fenómeno se explica por una observación en animales: la restricción calórica genera un aumento del consumo de drogas psicoactivas. Basándose en estos hallazgos, las guías de practica de cesación tabáquica (aun las más recientes) recomiendan no realizar simultáneamente dieta cuando se está dejando de fumar.

La actividad física como método para reducir la ganancia de peso ha sido pobremente evaluada, y los sustitutos de nicotina y el bupropion utilizados por 8 a 12 semanas en los tratamientos para dejar de fumar lograron retrasar, pero no evitar el aumento de peso post cesación tabáquica. También fue evaluada la fluoxetina, con resultados igualmente pobres.

Todo esto ha llevado a que las guías de práctica recomienden "asumir" la ganancia de peso post cesación como parte del tratamiento, más que a "combatirla".

Los programas de cesación tabáquico han mostrado que las mujeres son un grupo particularmente preocupado por el aumento de peso, lo cual constituye una barrera para intentar dejar de fumar y un predictor de recaída del tabaquismo. Por eso, varios autores han sugerido la necesidad de considerar, en la subpoblación de pacientes particularmente preocupada por el aumento de peso, la implementación simultanea de algún tratamiento que facilite el control del peso a fin de mejorar el éxito en las tasas de cesación.

\section{Resumen de la evidencia}

Cita 1:

Objetivo: determinar si la intervención dirigida a prevenir el aumento de peso, mejora la tasa de cesación tabáquica.

Diseño: Estudio aleatorizado, controlado, abierto, 16 semanas, seguimiento un año.

Lugar: clínica de obesidad. Estocolmo, Suecia.

Sujetos: 287 mujeres (30 - 60 años), BMI (23 - 31) tabaquistas de $=10$ cigarrillos/día, quienes hubieran recaído en un intento previo de dejar de fumar a causa del aumento de peso.

Intervención: tratamiento estándar para dejar de fumar con chicles de nicotina más tratamiento para bajar de peso: terapia conductual (11 sesiones de 45 minutos) y dieta intermitente severamente hipocalórica (Nutrilent $\AA^{\circledR}$ ) $700 \mathrm{kcal} /$ día aproximadamente. El grupo control recibió igual intervención excepto la dieta.

Medición de resultado principal: tasa de cesación tabáquica al año.

Medición de resultados secundarios: variación de peso al final del tratamiento y al año.

Resultados: ver tabla

Conclusiones: La combinación de un tratamiento para dejar de fumar con una intervención dirigida a controlar el peso en mujeres de edad media, mejoró la tasa de cesación tabáquica al año y ayudó a controlar el peso en el periodo inicial.

\section{Cita 2:}

Objetivo: determinar si el ejercicio, aumenta la tasa de cese tabáquico.

Diseño: Estudio aleatorizado de 12 semanas de duración, controlado, abierto, seguimiento a un año.

Lugar: Miriam Hospital, Brown University, EE.UU.

Sujetos: 281 mujeres sedentarias, (18 - 65 años), tabaquistas de $=10$ cigarrillos / $\mathrm{d}$ ía, quienes estuvieran interesadas en dejar de 
fumar y reducir la ganancia de peso posterior.

Intervención: todas las pacientes realizaron 12 sesiones de tratamiento cognitivo-conductual para dejar de fumar y mantener el peso. El grupo intervención realizó ters clases semanales de gimnasia aeróbica intensa de una hora de duración durante ocho semanas. El grupo control recibió igual cantidad de horas de intervención con actividades de lectura, videos y discusiones sobre hábitos saludables y prevención.

Medición de resultado principal: tasa de cesación tabáquica al final del tratamiento a los 3 y 12 meses.

Medición de resultados secundarios: variación de peso al final del tratamiento y al año.

Resultados: ver tabla

Conclusiones: el ejercicio aeróbico intenso, como parte de un tratamiento para dejar de fumar mejoró la tasa de abstinencia al año. El aumento de peso fue menor en el grupo intervención que en el grupo control al final del tratamiento, pero al año no hubo diferencias significativas en el peso entre ambos grupos.

\section{Cita 3:}

Objetivo: Evaluar la eficacia del bupropion en la prevención de la recaída del tabaquismo.

Diseño: Estudio aleatorizado doble ciego, controlado, seguimiento a dos años.

Lugar: Multicéntrico, EE.UU.

Sujetos: 784 pacientes motivados para dejar de fumar, tabaquistas de $=15$ cigarrillos/día.

Intervención: Luego de recibir siete semanas de tratamiento abierto con $300 \mathrm{mg}$ de bupropion, aquéllos que permanecieron abstinentes fueron randomizados a bupropion $300 \mathrm{mg}$ o placebo durante un año. Luego de finalizar el año de tratamiento fueron seguidos por un año más, sin recibir medicación. Durante los dos años de seguimiento fueron entrevistados mensualmente y se les realizaba consejo antitabáquico en cada consulta.

Medición de resultado principal: tasa de cesación tabáquica al año y tiempo a la recaída.

Medición de resultados secundarios: variación de peso al final del tratamiento, al año y a los dos años, y tasa de abstinencia continua a los dos años.

Resultados: ver tabla

Conclusiones: en personas que dejaron de fumar con bupropion durante 7 semanas de tratamiento, la terapia con bupropion por 12 meses mejoró la tasa de cesación al año, retrasó el tiempo a la recaída y disminuyó la ganancia de peso a los dos años.

\section{Evaluación critica de la literatura}

Los tres estudios seleccionados cumplen con criterios de una aceptable validez interna*:

- Los pacientes fueron correctamente aleatorizados

- El análisis fue hecho por intención de tratar*

- La valoración del resultado principal "cesación tabáquica" siempre tuvo un correlato bioquímico.

- El tiempo de seguimiento fue adecuado.

- Los grupos "intervención" y "control" fueron razonablemente homogéneos

- La ceguera es imposible en los 2 primeros estudios: no se puede ser ciego a una dieta hipocalórica y a un programa de actividad física.

- Las pérdidas al seguimiento fueron aceptables.

- En los estudios 1 y 2 se observan claros sesgos de selección, típicos de los ensayos en adicciones, en los que los pacientes son altamente seleccionados con tasas inhabituales de adherencia a las intervenciones.

\section{Validez externa* y aplicabilidad clínica}

Tanto el estudio 1 como el 2 incluyeron individuos similares a mi paciente (mujeres motivadas para dejar de fumar y con particular preocupación por el aumento de peso) por lo que sus conclusiones podrían ser aplicadas en mi situación.

Sin embargo la validez externa y su consecuente aplicabilidad clínica es cuestionable por varias razones: en el estudio 1, la intervención utilizada consistió en 11 encuentros de terapia conductual de 45 minutos, mas una dieta hipocalórica severa, más el tratamiento para dejar de fumar. $Y$ en el estudio 2, una similar cantidad de reuniones grupales, más la actividad física aeróbica intensa, de una hora de duración, tres veces por semana. Ambas intervenciones, particularmente la del estudio 1, parecen ser muy exigentes y difícilmente la población general se adheriría Por otra parte, los métodos resultan muy costosos y difíciles de implementar. Lamentablemente, los autores no reportan datos de costoefectividad de sus intervenciones, lo cual seria muy importante, antes de decidir implementar estrategias como éstas.

Lo que estos dos estudios aportan como novedoso e interesante, es que, es la primera vez que la dieta y la actividad física demuestran ser eficaces en la mejoría de las tasas de cesación tabáquica al

Tabla: Reporte de los resultados de los tres estudios 410

\begin{tabular}{|c|c|c|c|c|c|c|c|}
\hline & $\begin{array}{l}\text { Cesación tabáquica al aî́o } \\
(\%) \\
\text { Intervención vs. control } \\
\text { (valor de } p \text { ) }\end{array}$ & $\begin{array}{l}\text { RR } \\
\text { De cese tabáquico } \\
\text { al año } \\
\text { Interven. / control }\end{array}$ & RRA & NNT & $\begin{array}{l}\text { Cesación tabáquical } \\
2 \text { aíos }(\%) \\
\text { Intervención vs. control } \\
\text { (valor de p) }\end{array}$ & $\begin{array}{l}\text { Variación de peso al final del } \\
\text { tratamiento (kg.) } \\
\text { Intervención vs. control } \\
\text { (valor de p) }\end{array}$ & $\begin{array}{l}\text { Variación de peso al año/ dos } \\
\text { años (kg) } \\
\text { Intervención vs. control } \\
\text { (valor de p) }\end{array}$ \\
\hline Cita 1 & $\begin{array}{l}38 / 137(28 \%) \text { vs. } 24 / 150 \\
(16 \%) \\
(p<0,05)\end{array}$ & 1.73 & 0.12 & 8.5 & - & $\begin{array}{l}\text { Descenso de } 2.1 \mathrm{~kg} \text { (IC } 95 \% \\
2.9-1.3) \text { vs. aumento de } 1.6 \mathrm{~kg} \\
(\mathrm{IC} 95 \% 0.9-2.3) \\
(\mathrm{p}<0.001) .\end{array}$ & $\begin{array}{l}\text { No hubo diferencia significativa } \\
\text { en el peso: aumento de } 2.5 \mathrm{~kg} \\
(0.78-4.3) \text { y } 3.8 \mathrm{~kg}(2.5-5.1)(\mathrm{p} \\
=0,61) \text {. }\end{array}$ \\
\hline Cita 2 & $\begin{array}{l}11.9 \% \text { vs. } 5.4 \% \\
(p=0.05) .\end{array}$ & 2.2 & 0.06 & 15.4 & - & $\begin{array}{l}\text { Aumento de } 3.05+l-3.45 \text { vs. } \\
5.40+1-6.94 \\
(p=0.03) \text {. }\end{array}$ & $\begin{array}{l}\text { No hubo diferencia significativa } \\
\text { en el peso: } 8.9 \mathrm{~kg}+/-8.9 \mathrm{vs} \text {. } \\
5.76+-12.6\end{array}$ \\
\hline Cita 3 & $\begin{array}{l}55.1 \% \text { (IC: } 48.2,61.9) \text { vs. } \\
42.3 \% \text { (IC: } 35.5,49.2) \\
(p=0.008)\end{array}$ & 1.3 & 0.13 & 7.6 & $\begin{array}{l}\text { No hubo diferencia significativa } \\
(41.6 \% \text { vs. } 40 \%) p>0.05\end{array}$ & & $\begin{array}{l}\text { al año: } 3.8 \mathrm{~kg} \text { vs. } 5.6 \mathrm{~kg} \\
(p=0,002) \\
\text { a los dos años: } 4.1 \mathrm{~kg} \text { vs. } 5.4 \mathrm{~kg} \\
(p=0.016)\end{array}$ \\
\hline
\end{tabular}

RR (riesgo relativo) RRA (reducción de riesgo absoluto) NNT (numero necesario para tratar) fueron calculados a partir de los datos reportados en los estudios 
año y en el control de peso inicial post cesación, lo cual abre un horizonte de futuras investigaciones.

Un dato interesante en ambos estudios es que el beneficio en la disminución de la ganancia de peso, cesó al finalizar la intervención, sin embargo la tasa de cesación tabáquica persistió mas elevada en el grupo intervención incluso al año. Este fenómeno fue observado en ambos estudios lo cual hace suponer, que reducir la ganancia de peso en la fase inicial del cese tabáquico sería crucial en el pronóstico de la abstinencia a largo plazo.

Será necesario esperar a la aparición de nueva evidencia que evalué estas mismas intervenciones en el marco de programas menos intensivos, durante periodos más prolongados y mas realizables en la "vida real" así como también resultados de efectividad y costoefectividad de las mismas.

El estudio 3 es el primero que evaluó un tratamiento farmacológico por un periodo prolongado para la prevención de la recaída del tabaquismo. El uso prolongado de bupropion fue seguro, aumentó las tasas de abstinencia tabáquica y disminuyó significativamente el aumento de peso incluso después de dos años. Ahora bien, ¿es esta diferencia de peso clínicamente significativa? Entre el grupo bupropion y el grupo placebo la diferencia en $\mathrm{kg}$ fue de 1.8 y $1.3 \mathrm{~kg}$. para el primero y segundo año respectivamente. Tampoco disponemos de datos de costoefectividad y a priori el impacto clínico de esta diferencia absoluta de peso parece pequeño.

\section{Conclusiones}

En pacientes que desean dejar de fumar pero les preocupa la ganancia de peso se les puede proponer:

- Actividad física aeróbica tres veces por semana, ya que no sólo es eficaz para reducir el peso y mejorar la tasa de cese tabáquico sino también para favorecer al proceso de abandono del tabaco a través del control del estrés, del aumento de la sensación de bienestar, de la mejoría del humor y la prevención de la depresión. Además el ejercicio constituye, siempre, una alternativa saludable con beneficios probados por ejemplo, en prevención cardiovascular y en la calidad de vida.

- En segundo lugar consideraría la posibilidad de administrar bupropion por un tiempo prolongado, si la preocupación por el aumento de peso es muy importante, dado que es una droga segura y de eficacia probada para reducir la ganancia de peso (recordar que el beneficio en términos de ganancia de peso es pequeño).

- Respecto de la dieta hipocalórica severa, considero que debiéramos aguardar mayor evidencia antes de su recomendación debido a la difícil aplicabilidad clínica del método utilizado en el ensayo. Se puede aconsejar consumir frutas, verduras, líquidos y evitar dulces chocolates y otros sustitutos habituales del acto de fumar.

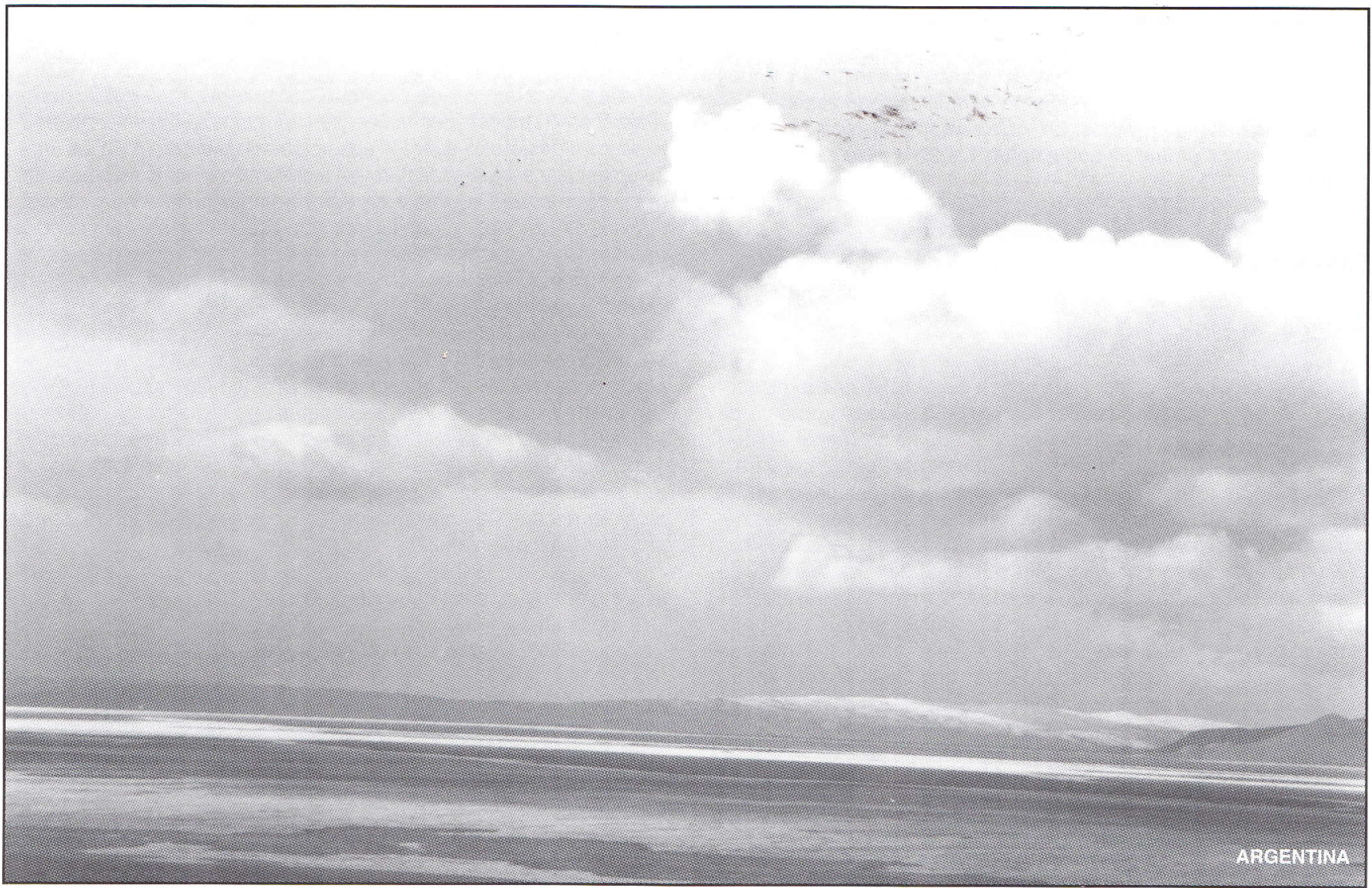

